\title{
Antimicrobial Profile of Acinetobacter spp. an Emerging Nosocomial Superbug
}

\author{
Nadakuduru Premanadham*, Madhurima and Siva Krishna \\ Narayana Medical College and Hospital, Nellore, South India \\ *Corresponding author
}

\begin{tabular}{|l|}
\hline Ke y w o r d s \\
Acinetobacter, \\
Antimicrobial \\
resistance, \\
Nosocomial \\
pathogen.
\end{tabular}

\section{Introduction}

Acinetobacter are Gram-negative Coccobacilli, strictly aerobic, non-motile, catalase positive, oxidase negative and lack pigmentation. (1) They are ubiquitous (2) free living saprophytes in soil and water.(3)

Up to $25 \%$ of healthy ambulatory adults exhibit cutaneous colonization by Acinetobacter and are the most common
Gram-negative bacteria carried on the skin of hospital personnel.(4) They are usually opportunistic pathogens reported to cause a number of outbreaks of nosocomial infections such as septicemia, pneumonia, wound sepsis, endocarditis, meningitis, urinary tract infections and peritonitis, (5) but their predominant role is in ventilator associated pneumonia (VAP), in intensive care units 
(ICUs).(1) Predisposing factors for Acinetobacter infections include the presence of prosthesis, endotracheal intubation, intravenous (I.V.) catheters and prior antibiotic therapy in a seriously ill-patient in hospital.(3) Such infections are often extremely difficult to treat because of widespread resistance to the major groups of antibiotics and long-term survival of bacteria in the hospital environment.(1)

Resistance to all known antibiotics has now emerged in Acinetobacter spp. with the majority of strains still being susceptible to carbapenems.(6) Multidrug-resistant (MDR) Acinetobacter infections are associated with increased time on mechanical ventilation, in the ICU and in the hospital.

Treatment options are severely limited; carbapenems and colistin are the agents of choice. More research and greater emphasis on the prevention of health-care associated transmission of MDR Acinetobacter infection are essential.(7)

The aim of this study was to isolate Acinetobacter species from clinical specimens and to study the antimicrobial susceptibility pattern of Acinetobacter isolates.

\section{Materials and Methods}

The study was carried out in the central laboratory of Microbiology Narayana Medical College Nellore South India from August 2015 to September 2016. Relevant clinical specimens sputum, blood, pus, urine were collected from patients by standard collection procedures. No specific exclusion criteria envisaged. Specimens were processed by standard microbiological techniques.(3). In Gram stain of direct smears Acinetobacter appeared as tiny, Gram-negative coccobacillary cells often appearing as diplococci.(5) All specimens were inoculated on $10 \%$ sheep blood agar and MacConkey agar and incubated at $37^{\circ} \mathrm{C}$ for $18-24$ h.(3) Colonies on blood agar were 0.5-2 mm diameter, translucent to opaque (never pigmented), convex and entire. On MacConkey agar a faint pink tint was produced.(5) Gram stain, catalase, oxidase and motility tests were performed. Acinetobacter are Gram-negative Coccobacilli, non-motile, strictly aerobic, catalase positive and oxidase negative. Rapid utilization of $10 \%$ glucose was seen with O-F medium. Acinetobacter species identification done. Acenetobactder baumanni 66(75.00\%) Acenetobacter lowfii 22(2.005\%).

Antimicrobial susceptibility testing(3) was performed by modified Kirby Bauer method(10) as per the Clinical and Laboratory Standards Institute guidelines.(11) Antibiotics tested were ampicillin, cephotoxime, cefizime, co-trimoxazole, ciprofloxacin, oflaxacin, gentamicin, amikasin, tygecyclin, amoxillin with clavulanic acid, ceforazolewith sulbactim, ticarcillin with clavulanic acid, piperacillin with tazobzctim, imipenem, cifriaxone and colistin.

\section{Results and Discussion}

In total, 88 Acinetobacter strains were isolated. Out of these 88Acinetobacter isolates, 36 isolates were from general wards and 52 were from ICU. Significantly higher percentage of Acinetobacter strains were found in ICU 56 (59.095) compared with general ward 36 (40.90\%). Acinetobacter infections were more common in males 64 $(72.73 \%)$ as compared with females 24 (27.27\%) (Table 1). Most infections are between 40 to 69 years as per table 3. The most common Acinetobacter isolates are from blood $45(51.14 \%)$ followed by pus 18 $(20.15 \%)$, urine $15(17.05 \%)$ and sputum (11.36\%) (Table 2). Most common Acinetobacter species isolated was 
Acinetobacter baumanni $66 \quad(75.00 \%)$, followed by Acenitobacter lowfii 22 (25.00\%). Imipenem was most sensitive drug $73(82.95 \%)$ followed by colistin 62 (70.45\%), Tigecyclin $59 \quad(67.05 \%)$, ciproflaxacin $55(62.50 \%)$, oflaxacin 54 (61.36\%), amikacin $53(60.23 \%)$ gentamycin $52(59.09 \%)$. Highest resistance is seen in ampicillin $70(79.35 \%)$ followed by cefixime 66 (75.00\%), cefriaxone $62 \quad(70.45 \%)$ amoxicillin + clavulanic acid 61(69.23\%), cephotoxime 57 (64.77\%), ticarcillin + clavulanic acid $54(61.35 \%)$ co-trimoxazole $49(55.68 \%)$, piperacillin + tazobactim 40 (45.45\%) (Table 4).

Acinetobacter spp. is Gram-negative Coccobacilli that contribute profoundly to the burden of modern medicine. Acinetobacter spp. is the second most commonly isolated non-fermenter in human specimens (after Pseudomonas aeruginosa). They rank fourth (after P. aeruginosa, Staphylococcus aureus and Klebsiella pneumoniae) among the most frequent hospital acquired infectious agents. (12) Acinetobacter spp. have emerged as a cause of ICUs infection. Multiresistant Acinetobacter spp. has become established as "alert" pathogens, particularly in ICUs and is associated with outbreaks of infection. (13) Their ubiquitous nature in the ICU environment and inadequate infection control practice has continuously raised the incidence of Acinetobacter infections over the past two decades. The understanding and recognition of Acinetobacter infections in the ICU is critically needed. (14)
In our study, a total number 88 Acinetobacter strains were isolated from processed clinical specimens. Houang et al., (15) reported a total of $1.32 \%$. Patients in ICU are sicker and require more invasive monitoring and therapeutic procedures to survive. ICU environmental contamination appears to be another important source of Acinetobacter infection. (14) The development of ICUacquired infections is strongly related to prolonged ICU stay and is associated with worse outcomes including increased morbidity and mortality.(18) In the present study, most common infections septicemias (blood 45-51.14\%) followed by wound infections (pus 18-20.25\%) pneumonia (sputum 20-11.36\%) urinary tract infections (urine 15-17.05\%). Joshi et al., (19) reported that 27.5 wound infections were caused by Acinetobacter. Acinetobacter ICU-acquired infections during the last decade represent a growing concern among clinicians and researchers. These infections most frequently involve the respiratory tract of intubated patients.(18)

In the present study, Acinetobacter infections were more common in males $64(72.73 \%)$ as compared with females 24 (27.27\%). This may be due to the fact that the males report more frequently to the hospitals compared with females. Prashanth and Badrinath(16) reported the infections to be more common in males $(58.00 \%)$ compared with females (42.00\%). Joshi et al., (19) reported 50.20\% infection in males.

Table.1 Age wise distribution of Acenitobacter spp

\begin{tabular}{|lcc|}
\hline TOTAL MALE & 64 & 72.73 \\
\hline TOTAL FEMALE & 24 & $27.27 \%$ \\
\hline TOTAL & 88 & $100 \%$ \\
\hline
\end{tabular}


Table.2 Various clinical samples of Acinetobacter spp

\begin{tabular}{|c|c|c|}
\hline Sample & Positive & $\mathbf{\%}$ \\
\hline BLOOD & 45 & $51.14 \%$ \\
\hline PUS & 18 & $20.45 \%$ \\
\hline URINE & 15 & $17.05 \%$ \\
\hline SPUTAM & 10 & $11.36 \%$ \\
\hline & 88 & $100.00 \%$ \\
\hline
\end{tabular}

Table.3 Age wise distribution

\begin{tabular}{|l|l|c|c|c|c|c|c|c|}
\hline$<10$ & NO OF MALE & $\mathbf{3}$ & $\mathbf{3 . 4 1 \%}$ & NO OF FEMALE & $\mathbf{0}$ & $\mathbf{0 . 0 0 \%}$ & $\mathbf{3}$ & $\mathbf{3 . 4 1 \%}$ \\
\hline $\mathbf{1 0}$ TO 19 & NO OF MALE & $\mathbf{4}$ & $\mathbf{4 . 5 5 \%}$ & NO OF FEMALE & $\mathbf{2}$ & $\mathbf{2 . 2 7 \%}$ & $\mathbf{6}$ & $\mathbf{6 . 8 2} \%$ \\
\hline $\mathbf{2 0}$ TO 29 & NO OF MALE & $\mathbf{8}$ & $\mathbf{9 . 0 9 \%}$ & NO OF FEMALE & $\mathbf{4}$ & $\mathbf{4 . 5 5 \%}$ & $\mathbf{1 2}$ & $\mathbf{1 3 . 6 4 \%}$ \\
\hline $\mathbf{3 0}$ TO 39 & NO OF MALE & $\mathbf{9}$ & $\mathbf{1 0 . 2 3 \%}$ & NO OF FEMALE & $\mathbf{3}$ & $\mathbf{3 . 4 1 \%}$ & $\mathbf{1 2}$ & $\mathbf{1 3 . 6 4 \%}$ \\
\hline $\mathbf{4 0}$ TO 49 & NO OF MALE & $\mathbf{1 1}$ & $\mathbf{1 2 . 5 0 \%}$ & NO OF FEMALE & $\mathbf{7}$ & $\mathbf{7 . 9 5 \%}$ & $\mathbf{1 8}$ & $\mathbf{2 0 . 4 5 \%}$ \\
\hline $\mathbf{5 0}$ TO 59 & NO OF MALE & $\mathbf{1 2}$ & $\mathbf{1 3 . 6 4 \%}$ & NO OF FEMALE & $\mathbf{3}$ & $\mathbf{3 . 4 1 \%}$ & $\mathbf{1 5}$ & $\mathbf{1 7 . 0 5 \%}$ \\
\hline $\mathbf{6 0}$ TO 69 & NO OF MALE & $\mathbf{1 4}$ & $\mathbf{1 5 . 9 1 \%}$ & NO OF FEMALE & $\mathbf{3}$ & $\mathbf{3 . 4 1 \%}$ & $\mathbf{1 7}$ & $\mathbf{1 9 . 3 2 \%}$ \\
\hline$>70$ & NO OF MALE & $\mathbf{3}$ & $\mathbf{3 . 4 1 \%}$ & NO OF FEMALE & $\mathbf{2}$ & $\mathbf{2 . 2 7 \%}$ & $\mathbf{5}$ & $\mathbf{5 . 6 8 \%}$ \\
\hline & & 64 & $72.73 \%$ & & 24 & $27.27 \%$ & 88 & $100 \%$ \\
\hline
\end{tabular}

Table.4 Antimicrobial pattern

\begin{tabular}{|c|c|c|c|}
\hline Antimicrobial AgenT & NO OF Resistance/\% & NO OF Sensitivity/\% & TOTAL \\
\hline \multirow[t]{2}{*}{ Ampicillin } & 70 & 18 & 88 \\
\hline & $79.55 \%$ & $20.45 \%$ & $100.00 \%$ \\
\hline \multirow[t]{2}{*}{ Cephotaxime } & 57 & 31 & 88 \\
\hline & $64.77 \%$ & $35.23 \%$ & $100.00 \%$ \\
\hline \multirow[t]{2}{*}{ Cefizime } & 66 & 22 & 88 \\
\hline & $75.00 \%$ & $25.00 \%$ & $100.00 \%$ \\
\hline \multirow[t]{2}{*}{ Co-trimoxazole } & 49 & 39 & 88 \\
\hline & $55.68 \%$ & $44.32 \%$ & $100.00 \%$ \\
\hline \multirow[t]{2}{*}{ Ciproflaxacin } & 33 & 55 & 88 \\
\hline & $37.50 \%$ & $62.50 \%$ & $100.00 \%$ \\
\hline \multirow[t]{2}{*}{ Oflaxacin } & 34 & 54 & 88 \\
\hline & $38.64 \%$ & $61.36 \%$ & $100.00 \%$ \\
\hline \multirow[t]{2}{*}{ Gentamycin } & 36 & 52 & 88 \\
\hline & $40.91 \%$ & $59.09 \%$ & $100.00 \%$ \\
\hline \multirow[t]{2}{*}{ Amikacin } & 35 & 53 & 88 \\
\hline & $39.77 \%$ & $60.23 \%$ & $100.00 \%$ \\
\hline \multirow[t]{2}{*}{ Tigecyclin } & 29 & 59 & 88 \\
\hline & $32.95 \%$ & $67.05 \%$ & $100.00 \%$ \\
\hline \multirow[t]{2}{*}{ Amoxicillin +clavulanic } & 61 & 27 & 88 \\
\hline & $69.32 \%$ & $30.68 \%$ & $100.00 \%$ \\
\hline \multirow[t]{2}{*}{ Ceperazone+sulbactim } & 33 & 55 & 88 \\
\hline & $37.50 \%$ & $62.50 \%$ & $100.00 \%$ \\
\hline \multirow[t]{2}{*}{ Tecarcillin +clavulanicacid } & 54 & 34 & 88 \\
\hline & $61.36 \%$ & $38.64 \%$ & $100.00 \%$ \\
\hline \multirow[t]{2}{*}{ Piparacillin+tzobactam } & 40 & 48 & 88 \\
\hline & $45.45 \%$ & $54.55 \%$ & $100.00 \%$ \\
\hline \multirow[t]{2}{*}{ Imepenium } & 15 & 73 & 88 \\
\hline & $17.05 \%$ & $82.95 \%$ & $100.00 \%$ \\
\hline \multirow[t]{2}{*}{ Cefriaxone } & 62 & 26 & 88 \\
\hline & $70.45 \%$ & $29.55 \%$ & $100.00 \%$ \\
\hline \multirow[t]{2}{*}{ Colistin } & 26 & 62 & 88 \\
\hline & $29.55 \%$ & $70.45 \%$ & $100.00 \%$ \\
\hline
\end{tabular}


Currently at least 31 Acinetobacter genomospecies have been described. Acinetobacter johnsonii, Acinetobacter lwoffii and Acinetobacter radioresistant seem to be natural inhabitants of human skin and commensals in human oropharynx and vagina.(5) The digestive tract of patients within ICUs often serve as reservoirs for multiresistant A. baumannii strains involved in hospital outbreaks.(2) The most common site for A. baumannii infection is the respiratory tract and the most common manifestation is VAP and bloodstream infections. A. lwoffii has been more commonly associated with meningitis, $A$. junii rarely causes ocular infection and bacteremia.(5) In our study, out of 88 Acinetobacter isolates, A. baumannii 66(75.00\%) was the most common species to cause Acinetobacter infection followed by acenitobacter lowfii 22(25.00\%) from 140 Acinetobacter isolates. Joshi et al., (19) isolated $70.00 \%$ A. baumannii, $1.40 \%$ Acinetobacter calcoaceticus, $\quad 6.40 \%$ Acinetobacter haemolyticus, $8.60 \%$ A. junii and $1.40 \%$ A. johnsonii. Prashanth and Badrinath(16) isolated $71.42 \%$ A. baumannii, $10.02 \%$ A. lwoffii, $4.08 \%$ A. haemolyticus and $2.04 \%$ strains of $A$. junii.

As noted by the Infectious Disease Society of America, Acinetobacter is "a prime example of mismatch between unmet medical need and the current antimicrobial research and development pipeline." Acinetobacter spp. are notorious for their ability to acquire antibiotic resistance.(22) Antimicrobial resistance among Acinetobacter spp. has increased substantially in the past decade and has created a major public health dilemma. The most potent antibiotic drug class currently available are the carbapenems, but resistant strains have emerged.(7) We have studied the antimicrobial resistance pattern among Acinetobacter isolates by Kirby-Bauer disc diffusion method. In our study, Acinetobacter isolates showed resistance to most of the antibiotics available. Acinetobacter spp. is universally resistant to penicillin, ampicillin and cephalothin. Various susceptibility to second and third generation cephalosporins have been reported.(5) Acinetobacter species possess a wide array of $\beta$-lactamases that hydrolyze and confer resistance to penicillins, cephalosporins and carbapenems. AmpC cephalosporinases are chromosomally encoded and confer resistance to broadspectrum cephalosporins. Class D oxacillinhydrolyzing-type enzymes, Class B metallo $\beta$ lactamases (MBLs), hydrolyze a broad array of antimicrobial agents, including carbapenems. Increasing antimicrobial resistance leaves few therapeutic options for MDR Acinetobacter infection. In our study, Imipenem was most sensitive drug 73 $(82.95 \%)$ followed by colistin $62(70.45 \%)$, Tygycyclin 59 (67.05\%), cproflaxacin 55 $(62.50 \%)$, oflaxacin $54(61.36 \%)$, amikacin $53(60.23 \%)$ gentamycin 52(59.09\%). Highest resistance is seen in ampicillin. 70(79.35\%) followed by cefixime $66(75.00 \%)$, cefriaxone $62(70.45 \%)$ amoxicillin + clavulanic acid 61 (69.23\%), cephotoxime 57 (64.77\%), ticarcillin + clavulanic acid $54(61.35 \%)$ cotrimoxazole $49(55.68 \%)$, piperacillin + tazobactim 40 (45.45\%) (Table 4).

Sinha et al., (23) reported 35.00\% Imipenem resistant Acinetobacter. Lee et al.,(24) reported $21.18 \%$ Corbella et al.,(25) reported $36.00 \%$ carbapenem resistant A. baumannii from the patients admitted to ICU.

Acinetobacter are the "superbugs" of the modern hospital environment causing significant proportion of infections in specific patient populations, especially in critically-ill patients in the ICU. As ubiquitous organisms (fortunately of low virulence), with few requirements for growth and survival, Acinetobacter spp. are prone to persist indefinitely in the hospital environment and to 
cause infections periodically when iatrogenic factors are present, i.e., overuse of broad spectrum antibiotics and high-risk patients. This situation, together with the fact that Acinetobacter isolates have inherent and/or easily acquired mechanisms of resistance against many of the available antimicrobial agents, makes this pathogen one of the most significant microbial challenges of the current era. Antibiotic resistance is attributed to production of extended spectrum betalactamase, MBL, loss of outer membrane proteins, efflux pumps and biofilm formation. Are there ways to control or limit the spread of these multiresistant strains? Is it still possible to treat Acinetobacter infections? First, it is necessary to improve microbiological techniques for early and more accurate identification and laboratory vigilance to prevent inappropriate empirical treatment.

Second, newer strategies for antibiotic use should be employed to reduce selection pressure, including more frequent rotation of antibiotic groups or sequential use of antibiotic classes. The development of totally new antibiotics with novel bacterial molecular target sites may constitute therapeutic alternatives within the next few years. Nevertheless, continued surveillance of prevalent organisms in ICUs, combined with preventive measures (e.g., isolation precautions, hand disinfection, efficient sterilization of instruments) remains absolutely essential in efforts to prevent or limit the spread of Acinetobacter infection. Continued awareness to maintain good housekeeping, control of the environment including equipment decontamination, strict attention to hand washing, isolation procedures and control of antibiotic usage, especially in high-risk areas, appear most likely measures to control the spread of Acinetobacter spp. in hospitals.

\section{References}

1. Bergogne-Bérézin E, Towner KJ. Acinetobacter spp. as nosocomial pathogens: Microbiological, clinical, and epidemiological features. Clin Microbiol Rev. 1996; 9:148-65.

2. Riley W. Acinetobacter and Moraxella. In: Borriello SP, Murray PR, Funke G, editors. Topley and Wilson's Microbiology and Microbial Infections: Bacteriology. 10th ed. Vol. 2. London: Hodder Arnold Publication; 2005. pp. 1301-11.

3. Collee JG, Fraser AG, Marmion BP, Simmons A. 14th edn. New York: Churchill-Livingstone; 1999. Mackie and McCartney Practical Medical Microbiology.

4. Allen DM, Hartman BJ. Acinetobacter species. In: Mandell GL, Bennett JE, Dolin $\mathrm{R}$, editors. Principles and Practice of Infectious Diseases. 5th ed. Vol. 2. Philadelphia: Churchill Livingstone; 2000. pp. 2239-44.

5. Koneman EW, Allen SD, Jande WM, Schreckenberger PC, Winn WC., Jr. 6th ed. Philadelphia: Lippincott Williams and Wilkins; 2006. Koneman's Colour Atlas and Textbook of Diagnostic Microbiology.

6. Peleg AY, Seifert H, Paterson DL. Acinetobacter baumannii: Emergence of a successful pathogen. Clin Microbiol Rev. 2008; 21: 538-82.

7. Maragakis LL, Perl TM. Acinetobacter baumannii: Epidemiology, antimicrobial resistance, and treatment options. Clin Infect Dis. 2008;46:1254-63. (PubMed)

8. Bauer AW, Kirby WM, Sherris JC, Turck M. Antibiotic susceptibility testing by a standardized single disk method. Am J Clin Pathol. 1966; 45: 493-6.

9. Wayne, PA, USA: CLSI; 2008. Clinical and Laboratory Standard Institute. Performance Standard for Antimicrobial Susceptibility Testing; Eighteenth Informational Supplement; M100-S18.

10. Shete VB, Ghadage DP, Muley VA, Bhore AV. Acinetobacter septicemia in neonates admitted to intensive care units. J Lab Physicians. 2009; 1:73-6. 
11. Agodi A, Zarrilli R, Barchitta M, Anzaldi A, Di Popolo A, Mattaliano A, et al., Alert surveillance of intensive care unit-acquired Acinetobacter infections in a Sicilian Hospital. Clin Microbiol Infect. 2006; 12: 241-7.

12. Rungruanghiranya S, Somboonwit C, Kanchanapoom T. Acinetobacter infection in the intensive care unit. $\mathrm{J}$ Infect Dis Antimicrob Agents. 2005; 22: 77-92.

13. Houang ET, Chu YW, Leung CM, Chu KY, Berlau J, Ng KC, et al., Epidemiology and infection control implications of Acinetobacter spp. in Hong Kong. J Clin Microbiol. 2001; 39: 228-34.

14. Prashanth K, Badrinath S. Nosocomial infections due to Acinetobacter species: Clinical findings, risk and prognostic factors. Indian J Med Microbiol. 2006; 24: 39-44.

15. Patwardhan RB, Dhakephalkar PK, Niphadkar KB, Chopade BA. A study on nosocomial pathogens in ICU with special reference to multiresistant Acinetobacter baumannii harbouring multiple plasmids. Indian J Med Res. 2008; 128: 178-87.

16. Falagas ME, Karveli EA, Siempos II, Vardakas KZ. Acinetobacter infections: A growing threat for critically ill patients. Epidemiol Infect. 2008; 136: 1009-19.

17. Joshi SG, Litake GM, Satpute MG, Telang NV, Ghole VS, Niphadkar KB. Clinical and demographic features of infection caused by Acinetobacter species. Indian J Med Sci. 2006; 60: 351-60.

18. Bennani B, Selmani R, Mahmoud M, Nejjari C, Kanjaa N. Nosocomial pneumonia in mechanically ventilated patients: Prospective study in intensive care unit of Fez University Hospital. Saudi J Anaesth. 2008; 2: 46-51.

19. Coelho JM, Turton JF, Kaufmann ME, Glover J, Woodford N, Warner M, et al., Occurrence of carbapenem-resistant Acinetobacter baumannii clones at multiple hospitals in London and Southeast England. J Clin Microbiol. 2006; 44: 3623-7.

20. Sinha $M$, Srinivasa $H$, Macaden $R$. Antibiotic resistance profile and extended spectrum beta-lactamase (ESBL) production in Acinetobacter species. Indian J Med Res. 2007; 126:63-7.

21. Lee SO, Kim NJ, Choi SH, Hyong Kim T, Chung JW, Woo JH, et al., Risk factors for acquisition of imipenem-resistant Acinetobacter baumannii: A case-control study. Antimicrob Agents Chemother. 2004; 48: 224-8.

22. Corbella $\mathrm{X}$, Montero A, Pujol $\mathrm{M}$, Domínguez MA, Ayats J, Argerich MJ, et al., Emergence and rapid spread of carbapenem resistance during a large and sustained hospital outbreak of multiresistant Acinetobacter. J Clin Microbiol. 2000; 38: 4086-4095.

\section{How to cite this article:}

Nadakuduru Premanadham, Madhurima and Siva Krishna. 2017. Antimicrobial Profile of Acinetobacter spp. an Emerging Nosocomial Superbug. Int.J.Curr.Microbiol.App.Sci. 6(12): 2230-2236. doi: https://doi.org/10.20546/ijcmas.2017.612.257 